\title{
A review of human and sub-human primate toxicity of hexachlorobenzene*
}

\author{
John Jarrell ${ }^{1}$ and Ayhan Gocmen² \\ ${ }^{1}$ Department of Obstetrics and Gynecology, University of Calgary, $140329^{\text {th }}$ St. \\ N.W. Calgary, Alberta, Canada T2N 2T9; '2Department of Pediatrics, Hacettepe \\ University, Ankara, Turkey
}

\begin{abstract}
The inadvertent exposure of a large cohort of individuals to hexachlorobenzene (HCB) in the mid-1950s in southeastern Turkey remains an environmental disaster that continues to fascinate and provide interesting observations concerning this ubiquitous chemical. A brief history of the exposure is presented with particular reference to the initial presentation of Pembe Yara and Porphyria Cutanea Tarda and later to reproductive performance in humans and sub-human primates. Differential toxicity is present in the oocytes of monkeys, with more severe damage identified in primordial germ cells rather than in growing oocytes. However, this observation did not express itself in women, as there was no increased incidence of premature menopause among those exposed. Germ cell toxicity remains a possible outcome of exposure, however. The serum HCB levels measured in exposed individuals predicted the lifetime risk of spontaneous abortion, not only in exposed individuals but also in the control populations, indicating that a possible population effect is present.
\end{abstract}

\section{INTRODUCTION}

Hexachlorobenzene (HCB) is an important chemical with the potential for serious human harm following exposure. The first available studies identified it as one of the chlorinated organic derivatives of benzene with limited or no toxicity [1]. These studies did not recognize that intense lipid solubility prevented appropriate biological uptake of the chemical to measure toxicity. Based upon several studies of effectiveness and agricultural efficiency, the chemical was recommended for use as a fungicide [2-4]. Specifically, it was recommended for the treatment of Tilletia tritici, a wheat-based fungus. The first summary of reports on the toxicity of HCB appeared in 1950, describing liver damage, irritation of the respiratory system, and eye damage with the warning that the allowable concentration was $50 \mathrm{ppm}$, but that it should be considered dangerous [5]. By the mid-1970s the chemical had been generally banned for use as a fungicide on a worldwide basis, although there were exceptions to the banning.

Reports of infant deaths from a mysterious disease began to emerge in the mid-1950s from the Turkish provinces of Diyarbakir, Urfa, and Mardin. Although first thought to be a congenital form of porphyria - a condition unknown in Turkey-it was suspected that the condition was due to the treatment of a wheat grain with HCB [6]. HCB was used for the first time in 1954 in limited quantities and then in higher quantities in later years. The treated grain, or seed grain, was to have been planted by farmers for subsequent harvesting the following year. Some have indicated that food shortages resulted in the deployment of the grain to markets for direct distribution, although all reports clearly indicate the deployment was inadvertent [7]. In some cases the grain was identified by its red color, but other reports indicate that it was similar in appearance to other wheat grains and passed into a more general distribution through markets in larger urban areas [7].

*Lecture presented at the $4^{\text {th }}$ Congress of Toxicology in Developing Countries ( $4^{\text {th }}$ CTOX-DC), Antalya, Turkey, 6-10 November, 1999. Other presentations are published in this issue, pp. 973-1066. 
Cam, a dermatologist and director of the skin clinic in Diyarbakir should be recognized for his very substantial accomplishments in this episode. He described one unique disease, Pembe Yara; the clinical appearance, course of the disease, and its relationship to hexachlorobenzene and breast-feeding. He also correctly recognized Porphyria Cutanea Tarda in a country that normally sees little of the condition, described the clinical course, and made the connection to the HCB-treated wheat [6]. Initially he described a congenitally based porphyria, but soon identified the condition as being acquired, and due to ingestion of HCB-treated wheat [8].

Pembe Yara, or pink sore, was identified as a condition associated with specific changes in the skin. It occurred among children from infancy to about 4 years of age. The children developed weakness, diarrhea, and the skin rash. A skin biopsy showed there was a proliferation of papillae, hyperkeratosis, and acanthosis of the epidermis. In subepithelial tissue there was an infiltration of lymphocytes, histiocytes and giant cells with strange bodies. Additionally, there was a hyperplasia of the pilo-sebaceous follicles. There are no published photographs of the histology of these lesions so that the full understanding of the condition is not possible. Clinically, the condition was very serious with a mortality rate $>95 \%$. This condition occurred in infants who were breast fed from mothers who had either developed Porphyria Cutanea Tarda or had ingested the treated wheat grain.

Cam also recognized and described the initial cases of Porphyria Cutanea Tarda and made the similar relationship to HCB [8]. In this instance, children over the age of 4 years up to late adolescence were affected. They developed a serious form of blistering in the sun-exposed areas of the skin. These bullae grew and ruptured and caused serious irritation of the skin. The lesions were active during the summer and receded in the winter. Only sun-exposed areas were affected. Additional signs of toxicity included hyperpigmentation of the skin and hypertrichosis. These changes were found all over the body. Although again reported as a congenital condition, the reports of the occurrence in different ethnic groupings caused the investigators to recognize its acquired basis. There was a substantial excess of males affected by the condition. Not all members of a family developed the disease in spite of similar diets. This suggests there was some individual variation in susceptibility, as well as a threshold effect.

These findings were supported by additional studies in depth of individual children and adolescents [9]. These investigators studied 6 cases from the town of Bismil; 4 children, all male. They corroborated the findings of Cam, describing in detail the clinical and laboratory findings and published one microphotograph of a bullous lesion indicating the appearance of cutaneous lesions without inflammation. The subjects were found to have no measurable urinary porphobilinogen despite very large quantities of urinary and fecal uroporphyrin(I) and coproporphyrin(III), indicating the failure of the porphyrin metabolizing system. They were further able to define the hepatic pathology as advanced hydropic and granular degeneration of the parenchyma, increased numbers of mononuclear cells in the capillaries, and the presence of a yellow pigment in the parenchymal cells. They were able to rule out heredity as a cause of the disease based upon the ethnic origins of the subjects [9].

Overall, the extent of the exposure in the population is unknown although it has been estimated that approximately 4-5000 individuals developed clinical porphyria. It is not known how many individuals developed Pembe Yara, but some have reported that some of the villages were without any children under the age of 5 years between 1955 and 1960 [7].

The dose that was required for the development of the conditions is not known. The fungicide was applied in a $0.2 \%$ concentration along with mercurous and mercuric chloride that had been used in previous years. Subsequent to the elimination of the HCB-treated wheat, patients began to improve and in Cam's reports, he was able to indicate there were limited case presentations after 1964 [10].

The actual association of the chemical with the disease was made stronger through the investigations of Ockner and Schmid (ref. 11] who demonstrated that a diet of $0.2 \%$ HCB in male rats could induce increased excretion of porphyrins and porphyrin precursors although there was no measurable uroporphyrin. Evaluation of the liver indicated hepatocellular degeneration. The condition was noted to be both reversible and apparently irreversible with the withdrawal of the HCB from the diet. These

(C) 2000 IUPAC, Pure and Applied Chemistry 72, 1015-1021 
findings provided confirmation that $\mathrm{HCB}$ was the cause of the condition in southeastern Turkey and eliminated the other possibilities of heredity and other pesticides previously used in the region [11].

A proportion of the exposed population has been followed by several investigators for the longterm outcomes of this event [10,12-19]. It is apparent that these subjects have continued to display long-lasting effects of the exposure to a variable degree. The subjects have had serious neurological, dermatological, and musculoskeletal abnormalities. Several reports have indicated that chelation therapy was of benefit in the eradication of the disease, although over time, many patients stopped having the summer-induced episodes of dermatological illness [20].

Recently, another population of severely exposed individuals has been identified in Flix, Catalonia, Spain. The exposure is primarily due to very high air levels $\left(35 \mathrm{ng} / \mathrm{m}^{3}\right)$. Despite having extremely high levels of serum $\mathrm{HCB}$, ranging up to $1616 \mathrm{ng} / \mathrm{mL}$, there were no alterations in porphyrin metabolism. These findings indicate there is a difference in individual susceptibility to the development of porphyria [21]. Adverse effects on reproductive function have not, however, been reported in women despite high serum HCB levels in a large cross-sectional study undertaken in Catalonia, Spain [22]. This study measured the prevalence of women who declare they had a spontaneous abortion and compared this prevalence to the findings of another study [23]. As the prevalence was within the range of the other study, the authors posited there was no effect of HCB [23].

\section{THE BASIS FOR REPRODUCTIVE INVESTIGATIONS}

There were no obvious problems associated with reproduction in the follow-up studies of those subjects that developed porphyria [24]. Despite these reassurances, animal investigations have revealed some intriguing outcomes of $\mathrm{HCB}$ exposure. The administration of ${ }^{14} \mathrm{C}$ labelled $\mathrm{HCB}$ in high specific activity followed by whole carcass autoradiography isolated HCB in certain endocrine tissues, particularly the ovary in a number of fish and fowl species [25-28]. Such a finding is no doubt due to the high lipophilicity and uptake of HCB in steroid-producing cells of the ovary and yolk sac and adrenal.

Further, Iatropoulos [29] administered the chemical in very high concentrations to Rhesus monkeys. The animals received from 8 to $128 \mathrm{mg} / \mathrm{kg} / \mathrm{day}$ for 60 days. They became quite ill, anorexic, and lost weight. As well as developing a severe wasting illness that included severe hepatic necrosis, fibrosis, and degeneration, there were very marked changes in the ovarian tissues. There was a severe destruction of the primordial germ cells of the ovary with an estimated $80 \%$ reduction in number of primary follicles. There were changes in the germinal epithelium that progressed from cuboidal to develop into a tall pseudostratified appearance.

There was interest in Canada because HCB was identified in human ovarian follicular fluid. In a study to determine the contamination of the ovarian follicle at the time of oocyte maturity, the use of the new reproductive technologies was utilized. Seventy-two patients undergoing in vitro fertilization in three centers in Canada (Hamilton, Halifax, and Vancouver) were studied. HCB was found as a common isolate in the follicular fluid and serum and the concentrations differed between the serum and follicular fluid and between the centers [30]. In addition to HCB, there were other chlorinated organic chemicals: a-chlordane, dichlorochlorophenylethylene (DDE), heptachlor epoxide-oxychlordane, and polychlorinated biphenyls. Although there was no significant correlation between the serum and the follicular fluid concentrations for HCB, such a relationship was indeed found to be highly significant for DDE. The presence of HCB, unlike other chemicals, was associated with a more rapid early embryo development rate.

Preliminary experiments using cynomolgus monkeys were undertaken to explore the observations made by Iatropoulos [29]. Animals received 8, 24, and $32 \mathrm{mg} / \mathrm{kg} /$ day orally for several weeks until the animals began to lose weight. The histology of the primordial cells was similar in description and in extent to the findings of Iatropoulos [29] and suggested a process of lipid peroxidation. These studies were expanded to pursue the possibility that lower concentration of HCB that did not cause a generalized illness could also affect reproductive function [31]. Another research question was to determine if

(C) 2000 IUPAC, Pure and Applied Chemistry 72, 1015-1021 
the chemical could interfere with large oocyte development, fertilization, and early embryonic development in addition to the marked toxicity demonstrated in the primordial germ cells. Sixteen animals were divided into four groups, receiving $0,0.1,1.0,10.0 \mathrm{mg} / \mathrm{kg} / \mathrm{day}$ HCB orally. The animals were given vehicle for 12 weeks prior to dosing with HCB for an additional 12 weeks. They were then subjected to in vitro fertilization. Briefly, human menopausal gonadotropin and human chorionic gonadotropin were administered in order to initiate ovarian follicular hyperstimulation. When there was appropriate ovarian response, the animals were anaesthetized and oocytes were collected through direct dissection from the ovary and the follicles after laparotomy. The oocytes were then inseminated with approximately 50000 spermatozoa from a male monkey that had undergone electroejaculation. The oocytes were subsequently observed for fertilization and early embryonic development. At the time of sacrifice the ovaries were removed and evaluated for histology and ultrastructure. Under these circumstances the animals remained healthy and did not lose weight.

The major observation was the apparent disconnection between the toxic effects on the primordial germ cells and the fully mature oocytes. Whereas there was a destruction of the primordial cells at high doses as determined by direct counting (high dose) and by ultrastructural evidence of lipid peroxidation (low dose), there were no measurable changes in large oocytes used during in vitro fertilization. These latter oocytes not only looked normal, but they underwent fertilization and early embryonic development at a rate that was comparable to control. The primordial cells had the same appearances as those treated with very high concentrations of HCB, that of lipid peroxidation, although this has never been confirmed. The normal function of larger follicles suggested that there may be a specific process of relative protection for larger follicles and oocytes. These findings also suggested that there may be continual destruction of the bank of primordial germ cells in women despite what would appear to be normal ovarian function from the perspective of large ovulatory follicles.

Another finding was the presence of ultrastructural abnormalities in the surface epithelium of the ovary [32]. This had been reported in the original work of Iatropoulos as a hyperplasia through light microscopy. In the current studies a distinct abnormality of the surface epithelium is readily identified at low dosages $[29,33]$.

A recent reproductive study in rats has demonstrated the same ovarian findings that were reported in monkeys; degenerative changes in the primordial follicles and alterations in the germinal epithelium [34]. The rats also had reduced estradiol and prolactin concentrations in the serum and decreased uterine nuclear estrogen receptors.

The significance of this finding is not certain at present although it is important to indicate these cells are the progenitors of ovarian epithelial cancer.

\section{RATIONALE FOR HUMAN INVESTIGATIONS}

Investigations in women were undertaken to test the hypothesis that HCB may induce premature ovarian failure or premature menopause in women. Because of the known exposure in women's ovarian follicles and the predilection for damage to primordial germ cells in sub-human primates, ovarian failure seemed a possibility. The normal function of larger follicles in the cynomolgus monkeys as defined by fertilization and cleavage, despite numeric loss and evidence of ultrastructural damage in primordial germ cells suggested that there may be a relevant clinical issue for women. It was possible that exposure to the HCB might have little effect through the transudate in the ovarian follicle at the termination of the ovulatory cycle. However, it might have a quiescent sub-clinical effect on the longevity of primordial follicles that would result in a normal ovulatory life-span and eventuate in a premature menopause because of the limited bank of committed germ cells.

The women who survived the Turkish HCB episode were identified as an appropriate group to evaluate for human reproductive outcomes. As the episode in Turkey occurred in 1955-60, a review undertaken in 1996 would allow an assessment of essentially the entire reproductive outcomes as well as the onset of natural and premature menopause.

(C) 2000 IUPAC, Pure and Applied Chemistry 72, 1015-1021 
Subjects were selected from the files of Prof. Ayhan Gocmen who has been following a cohort of patients with confirmed Porphyria Cutanea Tarda [35]. Forty-two subjects were selected on this basis (Group 1). In addition, 42 subjects were selected from the same region of Diyarbakir in southeastern Turkey who had no known exposure to the grain (Group 2) and 42 subjects were obtained from the capital, Ankara (Group 3). The control groups were selected to provide a comparative basis for the subjects in Group 1 with porphyria. In addition to the completion of reproductive questionnaires, FSH, estradiol and inhibin were measured in serum to assist with the identification of menopausal state. Blood was also taken for the measurement of HCB. Although the study was undertaken approximately 40 years after the episode, the half-life of HCB is thought to be extremely long because of the intense lipophilicity and low levels of metabolism.

Interestingly, the control subjects did not differ from the exposed group in the mean serum concentrations of HCB, although higher levels of HCB were identified in Group 1. The lack of a significant difference would suggest that 1) the HCB had been effectively eliminated from the exposed population, 2) there are other sources of HCB in Turkey, and 3) exposure was more widespread than the incidence of porphyria would suggest.

There were no cases of premature menopause in the entire study population and no differences among the groups in terms of the prevalence of natural menopause. There were no differences in age at menarche, increase in infertility, or stillbirths. There were very interesting findings in relation to spontaneous abortion. The prevalence of abortion in the entire study group was very high. When all subjects are reviewed it is apparent that approximately one half of the subjects reported an abortion rate greater than $20 \%$. However, the rate did not differ between groups. Because all groups had been exposed, the analysis of the relationship between HCB was done with all subjects. The serum HCB was highly predictive of abortions in all subjects $(\mathrm{p}=0.002)$.

These findings indicate that in this particular study group HCB was a remarkably enduring biological marker for a clinically relevant outcome. There was evident merit in the use of the serum HCB as a biological marker for the effect of abortion. The lack of effect on the establishment of premature menopause is of interest. This may be explained on the basis of species differences or alternatively the impact of the degree of oocyte destruction because HCB may have reduced over time.

The possible mechanisms of inducing abortion in this population of subjects are unknown. There are suggestions that altered steroidogenesis and or altered immune function may have a role. It has been shown that HCB reduces luteal phase serum progesterone concentrations in the primates tested, although the mechanism of this finding is currently unknown [36,37]. More recently, there has been an interest in the possibility that $\mathrm{HCB}$, along with other chlorinated hydrocarbons, may be associated with altered immune function. Gerhard et al [38] have elaborated this finding to show that altered immune function tests were abnormal among some patients with abortion although there were no significant differences between groups of patients that were primary vs. secondary aborters, early or late aborters. Increasing concentrations of pentachlorophenol was associated with a reduced lymphocyte count, $\operatorname{IgG}$ levels, and lymphocyte stimulation. Gamma hexachlorocyclohexane was associated with increased levels of T-helper cells in secondary abortion and increased monocytes and Natural Killer cells in primary aborters. HCB was found to reduce T-suppressor cells and increase the T-helper/T-suppressor cell ratio [38].

\section{SUMMARY}

This unique environmental catastrophe has provided some much needed insight into our understanding of the evaluation of human reproductive outcomes in response to severe chemical exposures. Because of the assumed purity of the toxic spill in relation to other chlorinated organic chemicals, it has been possible to evaluate HCB exposure in humans with reference to important animal experiments. In some cases the results are similar, such as the reproductive effects of HCB on rats and monkeys. However, this has not extended to humans at least in terms of the severity of the ovarian lesions. The reason for this

(C) 2000 IUPAC, Pure and Applied Chemistry 72, 1015-1021 
discrepancy is unknown at present. The impact of HCB on humans requires further study, particularly in relation to immune mechanisms that may interfere with implantation and early embryonic and fetal development as well as the potential for effects on ovarian surface epithelium. HCB remains a priority chemical for evaluation in humans, particularly human reproduction. Long-term outcome studies are warranted in the areas of reproductive loss and ovarian cancer.

\section{REFERENCES}

1. G. T. J. Cameron. J. Pathol. Bacteriol. 44, 281-296 (1920).

2. M. Lansade. Parasitica 5, 1-4 (1949).

3. M. Lansade. Comptes rendus de l'Acadamie d'Agriculture de France (1948).

4. M. Lansade. Comptes-rendus de l'Acad d'Agriculture de France (1946).

5. H. Elkins. The Chemistry of Industrial Toxicology. John Wiley, New York (1950).

6. C. Cam. Annales de Dermatologie et de Syphiligraphie 87, 393-397 (1960).

7. R. Schmid. New Engl. J. Med. 263, 397-398 (1960).

8. C. N. Cam. JAMA 183, 90-93 (1963).

9. A. I. Cetingil and M. A. Ozen Blood 16, 1002-1011 (1960).

10. H. A. Peters, S. A. Johnson, S. Cam, Y. Muftu, S. Oral, T. Ergene, D. J. Cripps. Am. J. Med. Sci. 251, 314-322 (1966).

11. R. K. Ockner and R. Schmid. Nature 189, 499 (1961).

12. A. Gocmen, H. A. Peters, D. J. Cripps, G. T. Bryan, C. R. Morris. Biomed. Environ. Sci. 2, 36-43 (1989).

13. H. Peters, D. Cripps, A. Gocmen, G. Bryan, E. Erturk, C. Morris. Ann. NY Acad. Sci. 514, $183-$ 190 (1987).

14. A. Gocmen, H. A. Peters, D. J. Cripps, C. R. Morris, I. Dogramaci. IARC Sci. Publ. 77, 567-573 (1986).

15. D. J. Cripps, H. A. Peters, A. Gocmen, I. Dogramaci. Br. J. Dermatol. 111, 413-422 (1984).

16. H. A. Peters, A. Gocmen, D. J. Cripps, G. T. Bryan, I. Dogramaci. Arch. Neurol. 39, 744-749 (1982).

17. D. J. Cripps, A. Gocmen, H. A. Peters. Arch. Dermatol. 116, 46-50 (1980).

18. H. A. Peters. Fed. Proc. 35(12), 2400-2403 (1976)

19. H. A. Peters, S. A. Johnson, S. Cam, Y. Muftu, S. Oral, T. Ergene. Am. J. Med. Sci. 251, 314-322 (1966).

20. R. W. Lambrecht, E. Erturk, H. A. Peters, C. R. Morris, G. T. Bryan. IARC Sci. Publ. 77, 505-506 (1986).

21. C. Herrero, D. Ozalla, M. Sala, R. Otero, M. Santiago-Silva, M. LechaJ. To-Figueras, R. Deulofeu, J. M. Mascaro, J. Grimalt, J. Sunyer. Arch. Dermatol. 135, 400-404 (1999).

22. M. Sala, J. Sunyer, R. Otero, M. Santiago-Silva, D. Ozalla, C. Herrero, J. To-Figueras, M. Kogevinas, J. M. Anto, C. Camps, J. Grimalt. Arch. Environ. Health. 54, 102-109 (1999).

23. A. Correa, R. H. Gray, R. Cohen. Am. J. Epidemiol. 143, 707-717 (1996).

24. H. Peters, D. Cripps, A. Gocmen, G. Bryan, E. Erturk, C. Morris. Ann. NY Acad. Sci. 514, $183-$ 190 (1987).

25. K. Ingebrigtsen and J. E. Solbakken. J. Toxicol. Environ. Health 16, 197-205 (1985).

26. K. Ingebrigtsen and J. U. Skaare. J. Toxicol. Environ. Health 12, 309-316 (1983).

27. K. Ingebrigtsen and I. Nafstad. Acta Pharmacol. Toxicol. (Copenh.) 52, 254-260 (1983).

28. K. Ingebrigtsen, E. M. Brevik, I. Nafstad. J. Toxicol. Environ. Health 8, 845-856 (1981). 
29. M. J. Iatropoulos , W. Hobson, V. Knauf, H. P. Adams. Toxicol. Appl. Pharmacol. 37, 433-444 (1976).

30. J. F. Jarrell, D. Villeneuve, C. Franklin, S. Bartlett, W. Wrixon, J. Kohut, C. G. Zouves. CMAJ 148, 1321-1327 (1993).

31. J. F. Jarrell, A. McMahon, D. Villeneuve, C. Franklin, A. Singh, V. E. Valli, S. Bartlett. Reprod. Toxicol. 7, 41-47 (1993).

32. K. A. Babineau, A. Singh, J. F. Jarrell, D. C. Villeneuve. J. Submicrosc. Cytol. Pathol. 23, 457464 (1991).

33. A. C. Bourque, A. Singh, N. Lakhanpal, A. McMahon, W. G. Foster. Am. J. Vet. Res. 56, 16731677 (1995).

34. L. Alvarez A. Randi, P. Alvarez, G. Piroli, A. Chamson-Reig, V. Lux-Lantos, D. Kleiman de Oisarev. J. Appl. Toxicol. 20, 81-87 (2000).

35. J. Jarrell, A. Gocmen, W. Foster, R. Brant, S. Chan, M. Sevcik. Reprod. Toxicol. 12, 469-476 (1998).

36. W. G. Foster, A. McMahon, E. V. Younglai, J. F. Jarrell, P. Lecavalier. Reprod. Toxicol. 9, 541-548 (1995).

37. W. G. Foster, A. McMahon, D. C. Villeneuve, J. F. Jarrell. J. Appl. Toxicol. 12, $13-17$ (1992).

38. I. Gerhard, V. Daniel, S. Link, B. Monga, B. Runnebaum. Environ. Health. Perspect. 106, 675681 (1998).

(C) 2000 IUPAC, Pure and Applied Chemistry 72, 1015-1021 